\title{
A GAUSS PROGRAM FOR COMPUTING THE FOULKES-DAVIS TRACKING INDEX FOR POLYNOMIAL GROWTH CURVES
}

\author{
EMET D. SCHNEIDERMAN ${ }^{a}$, STEPHEN M. WILLIS ${ }^{a}$, CHARLES J. KOWALSKI ${ }^{b}$ and THOMAS \\ R. TEN HAVEc
}

\begin{abstract}
${ }^{a}$ Department of Oral and Maxillofacial Surgery, Baylor College of Dentistry, 3302 Gaston Avenue, Dallas, TX 75246, ${ }^{b}$ Department of Oral Biology and 'c Department of Biostatistics, The University of Michigan, Ann Arbor, MI 48109 (USA)
\end{abstract}

(Received June 1st, 1992)

(Accepted July 6th, 1992)

\begin{abstract}
We have previously published a GAUSS program for computing the Foulkes-Davis tracking index, $\gamma$, from a one-sample longitudinal data set when no assumptions were made concerning the structure of the individual growth curves (Schneiderman et al, Am J Hum Biol, 4 (1992) 417-420). In this paper we consider the computation of the Foulkes-Davis index assuming that each individual growth curve may be adequately represented by a polynomial function in time and a GAUSS program performing these computations is made available. As with the two other tracking indices we have described, $\gamma$ and $\kappa$ (Schneiderman et al., Am J Hum Biol, 2 (1990) 475-490), this one can be used to evaluate regularity in patterns of growth or adaptation. An example is presented where statural growth in the same three groups considered in the earlier papers are analyzed. The small disparities between these and the earlier results are discussed in view of the different assumptions of the models and the differences in how they operationalize the concept of tracking.
\end{abstract}

Key words: Longitudinal studies; Growth curves; Polynomials; PC program; Tracking

\section{Introduction}

In a recent paper [1] we described a tracking index for longitudinal observations due to Foulkes and Davis [2] which did not assume any particular structure for the growth curves. Such an index has the obvious advantage of being widely applicable, but sharper results may be obtained when it is reasonable to assume that the growth curves have some specified functional form. The purpose of the present paper is to extend the Foulkes-Davis (FD) tracking index to the situation in which the growth curves may be assumed to be polynomials (tests of whether or not this assumption is tenable are included) and to provide a GAUSS program which will do the associated computations. An example illustrating the technique and the use of the program is given. The results are compared with those obtained using the 'nonparametric' form of the FD index and the index based on the kappa statistic [3].

Correspondence to: Emet D. Schneiderman, Department of Oral and Maxillofacial Surgery, Baylor College of Dentistry, 3302 Gaston Avenue, Dallas, TX 75246, USA. 


\section{The Foulkes-Davis Tracking Index}

The FD tracking index is, simply, the probability, $\gamma$, that two randomly selected growth curves will not intersect (cross) over the time frame of the study. Thus the index is predicated on the notion of pairs of individuals maintaining relative rank within the response distribution as it changes over time, i.e. the idea that if a given individual is 'smaller' (larger) than another given individual at the first time of measurement, he/she tends to remain smaller (larger) as time progresses. Since $\gamma$ is a probability, $0 \leq \gamma \leq 1$. No tracking is said to occur if $\gamma<1 / 2$ and perfect tracking corresponds to $\gamma=1$. Intermediate values of $\gamma$ represent varying degrees of tracking; the higher the value of $\gamma$, the more the population tends to track. No tracking corresponds' to $\gamma<1 / 2$ for when the probability of two randomly chosen curves crossing is at least $1 / 2$, relative rank at any one point in time provides no information concerning relative rank at another. Perfect tracking is said to occur if $\gamma=1$ for then no two growth curves intersect, i.e. for every pair of individuals in the population, relative rank within the response distribution is maintained over time. Obviously, if all the growth curves in the population are parallel, $\gamma=1$. The converse, however, is not true since all that is required under the FD concept of tracking is that the curves do not intersect. There is no requirement that the curves 'behave similarly'. However, if $N$ is large, the growth curves would have to behave reasonably similarly in order not to intersect.

\section{Estimation of $\gamma$}

Given a longitudinal data set

$$
\underset{N \times T}{\mathbf{X}}=\left[\begin{array}{cccc}
x_{11} & x_{12} & \cdots & x_{1 T} \\
x_{21} & x_{22} & \cdots & x_{2 T} \\
\vdots & \vdots & \cdots & \vdots \\
x_{N 1} & x_{N 2} & \cdots & x_{N T}
\end{array}\right]=\left[\begin{array}{l}
\mathbf{x}_{1}^{\prime} \\
\mathbf{x}_{2}^{\prime} \\
\vdots \\
\mathbf{x}_{N}^{\prime}
\end{array}\right]
$$

where $x_{i j}$ denotes the value of the observation made on the $i$ th individual $(i=1$, $2, \ldots, N)$ at time $t_{j}(j=1,2, \ldots, T)$ and $\mathbf{x}_{i}$ is the $T \times 1$ vector of observations for the $i$ th individual, $\gamma$ is estimated by comparing the growth curves for each of the

$$
\left(\begin{array}{c}
N \\
2
\end{array}\right)=N(N-1) / 2 \text { pairs of individuals in the sample and taking } \hat{\gamma} \text { to be the propor- }
$$

tion of these which do not cross at any of the times of measurement. An explicit formula for this estimator is most conveniently given in terms of an indicator function: If $\boldsymbol{\Psi}_{i k}=1$ when the growth curves of individuals $i$ and $k$ do not cross and $\boldsymbol{\Psi}_{i k}=0$ when they do, then

$$
\hat{\gamma}=\sum_{i<k} \Psi_{i k} /\left(\begin{array}{c}
N \\
2
\end{array}\right)
$$


The restriction on the summation in Eqn. (2), $i<k$, is made so as not to count the comparison of individuals $i$ and $k$ twice.

In Eqn. (1), the observed values for individuals $i$ and $k$ were simply compared at each of the time points and $\Psi_{i k}$ was assigned the value 1 if individual $i$ was always (i.e. at every time point) at least as large ( $\geq$ ), or at most as small ( $\leq$ ), as individual $k$. No functional form for the growth curve was assumed. In the following section we show how $\gamma$ is estimated when a polynomial is fit to each individual's growth profile.

\section{Polynomial Growth Curves}

FD assume that the growth curve for the $i$ th individual is a polynomial in time $(t)$ of the same degree $(D)$ for each individual, viz.,

$$
x_{i}(t)=\tau_{i 1}+\tau_{i 2} t+\cdots+\tau_{i P t} D
$$

or, in matrix notation,

$$
x_{i}(t)=\mathbf{t}^{\prime} \tau_{i}
$$

where $\mathrm{t}^{\prime}=\left[1, t, \ldots, t^{D}\right]$ and $\tau_{i}$ is the $P \times 1(P=D+1)$ vector of regression coefficients for the $i$ th individual. In our program, $D$ is determined by a sequence of preliminary goodness-of-fit tests. We begin by testing $D=1$ and increment $D$, when necessary, until an acceptable fit is found using the user-specified level of significance for the tests [4]. FD make no assumptions concerning the structure of the covariance matrix, $\Sigma$, of the $\mathbf{x}_{i}$ (c.f. Ref. 4) and hence estimate the $\tau_{i}$ by

$$
\hat{\tau}_{i}=\left(\mathbf{W}^{\prime} \mathbf{S}^{-1} \mathbf{W}\right)^{-1} \mathbf{W}^{\prime} \mathbf{S}^{-1} \mathbf{x}_{i}
$$

where $\mathbf{S}$ is the $T \times T$ sample covariance matrix of the $\mathbf{x}_{i}$ and $\mathbf{W}$ the withinindividual (time) design matrix

$$
\mathbf{W}=\left[\begin{array}{cccc}
1 & t_{1} & \cdots & t_{T}^{D} \\
1 & t_{2} & \cdots & t_{2}^{D} \\
\cdot & \cdot & & \cdot \\
\cdot & \cdots & . \\
\cdot & \cdot & & \cdot \\
1 & t_{T} & \cdots & t_{T}^{D}
\end{array}\right]
$$

The times of measurement $t_{1}, t_{2}, \ldots, t_{T}$ need not be equally spaced, but are assumed to be the same for each individual.

Given this structure the indicator function $\Psi_{i k}$ is written

$$
\psi_{i k}=1 \text { if } \min _{t}\left\{\mathbf{t}^{\prime}\left(\hat{\tau}_{i}-\hat{\tau}_{k}\right)\right\} \geq 0 \text { or } \max _{t}\left\{\mathbf{t}^{\prime}\left(\hat{\tau}_{i}-\hat{\tau}_{k}\right)\right\} \leq 0
$$


and $\Psi_{i k}=0$ otherwise. This accomplishes the same thing as the original $\Psi_{i k}$, i.e. $\Psi_{i k}=1$ only when the growth curves for the two individuals do not cross during the observation period. Notice, e.g., if the smallest of the differences between individual $i$ values and individual $k$ values growth curves is non-negative, then all such differences are non-negative, i.e. the polynomial growth curve for individual $i$ value is always 'above' that of individual $k$ value.

Equation (6) is the FD method for computing the $\Psi_{i k}$. In practice the minimum and maximum values are obtained by evaluating $\mathrm{t}^{\prime}\left(\hat{\tau}_{i}-\hat{\tau}_{k}\right)$ at $t=t_{1}, t_{2}, \ldots, t_{T}$ and the $\boldsymbol{\Psi}_{i k}$ evaluated by comparing the smallest and largest of these values to zero. We do this slightly differently. We replace $\mathbf{X}$ by $\mathbf{X}^{*}$ where $\mathbf{X}^{*}$ contains the fitted values of the polynomials at times $t_{1}, t_{2}, \ldots, t_{T}$ and use the algorithm developed previously for our program which computes the non-parametric form of the FD index [1]. Rather than computing differences between growth curves and comparing these to zero, we compare the (fitted) growth curves directly. In this light it is clear that the FD polynomial tracking index is not really all that different from the non-parametric version. When polynomials are fit to the individual growth profiles we are, in essence, replacing the observations $\mathbf{X}$ by 'smoothed' values $\mathbf{X}^{*}$, the elements of $\mathbf{X}^{*}$ being the values of the individual polynomials at $t_{1}, t_{2}, \ldots, t_{T}$. Smoothing may eliminate 'minor' crossings (intersections which reflect random fluctuations and not the overall trend of growth).

In any event, having computed the $\boldsymbol{\Psi}_{i k}$ for every pair of individuals ( $\boldsymbol{\Psi}_{i k}=\Psi_{k i}$ ) we again [1] estimate $\gamma$ by

$$
\hat{\gamma}=\frac{1}{N} \sum_{i=1}^{N} \psi_{i}
$$

where

$$
\psi_{i}=\frac{1}{N-1} \sum_{\substack{k=1 \\ k \neq i}}^{N} \psi_{i k}
$$

The S.E of $\hat{\gamma}$ is estimated by

$$
\text { S.E. }(\hat{\gamma})=\sqrt{\frac{\operatorname{Var}\left(\psi_{i}\right)}{N}}
$$

and, for large samples, an approximate $95 \%$ confidence interval for $\gamma$ is

$$
\hat{\gamma} \pm 2 \text { S.E. }(\hat{\gamma})
$$

Note that $\Psi_{i}$ is a measure of how well the $i$ th individual tracks: It is the proportion of times that the ith individual's growth curve does not intersect the other $N-1$ growth curves. Below we describe our program, apply it to three data sets considered in earlier publications and compare the results. 


\section{The Program}

The program is called FDTRK2 and is invoked by issuing the command GAUSSRUN FDTRK2. A variety of options are made available to the user by means of an interactive screen menu. Several data sets can be evaluated with a single run. Data sets can be input using either ASCII format or GAUSS format. Data collected at unequal time points can be evaluated as readily as those collected at equally spaced intervals. The user also selects the level of significance ( $\alpha$ level) to be used in the sequential goodness-of-fit tests for determining $D$, the degree of the polynomial used to fit the growth curves.

In terms of the output, the program first prints the usual descriptive statistics and then the results of the step-up goodness-of-fit tests, i.e. F-statistics and the corresponding $\mathrm{p}$-values. The polynomial regression coefficients are also generated for each of the $N$ individuals. Finally, the indicator matrix, the value of the FD index, the length of the half-interval and $95 \%$ confidence interval are given. The graphical output includes a plot of the individual growth profiles and a plot of the best-fit polynomials. If more than one data set is being analyzed, the results for each are produced sequentially. Details concerning how to obtain a copy of the program, as well as hardware and software requirements are provided in the Appendix.

\section{Examples}

We consider statural growth in three samples of children living in Guatemala which were studied in depth in Bogin et al. [5]. The children comprising these samples differ in socioeconomic status (SES) and ethnicity: One is of low SES Mayan Indian children $\left(G_{1}\right)$; the second is of low SES Ladino children $\left(G_{2}\right)$; the third is of high SES Ladino children $\left(\mathrm{G}_{3}\right)$. Bogin et al. [5] showed, among other things, that SES-related deficits in growth are cumulative during childhood and early adolescence; and that childhood growth deficits of low SES children are likely to carry over into adulthood. The prominence given these results reflects the importance that the concept of tracking commands in describing the growth patterns of several populations. These investigators did not, however, formally quantify and/or compare the tracking behavior within and between these populations. In an earlier paper [6] we did this for body height in the threc groups of 20 subjects each using the kappa statistic, testing the hypothesis that

$$
H_{0}: \kappa_{1}=\kappa_{2}=\kappa_{3}
$$

We found that

$$
\begin{aligned}
& \kappa_{1}=0.74668, \text { S.E. }=0.04116\left(P_{\mathrm{o}}=0.83333\right) \\
& \kappa_{2}=0.70370, \text { S.E. }=0.04092\left(P_{\mathrm{o}}=0.80333\right) \\
& \kappa_{3}=0.72796, \text { S.E. }=0.04098\left(P_{\mathrm{o}}=0.82000\right)
\end{aligned}
$$

The values $P_{\mathrm{o}}$ are the uncorrected (for chance) tracking indices in the three groups 
TABLE I

THE VALUES OF $\kappa$, FD I AND FD II AND THE HALF-WIDTHS OF THE CORRESPONDING CONFIDENCE INTERVALS FOR THE THREE SAMPLES

$G_{1}$, low SES Mayan children; $G_{2}$, low SES Ladino children; $G_{3}$, high SES Ladino children.

\begin{tabular}{llll}
\hline & $\kappa$ & FD I & FD II \\
\hline$G_{1}$ & $0.74668 \pm 0.08232$ & $0.85789 \pm 0.034237$ & $0.96842 \pm 0.014081$ \\
$G_{2}$ & $0.70370 \pm 0.08184$ & $0.73158 \pm 0.051171$ & $0.95789 \pm 0.021053$ \\
$G_{3}$ & $0.72796 \pm 0.08196$ & $0.81053 \pm 0.035327$ & $0.92632 \pm 0.020774$ \\
\hline
\end{tabular}

$[1,6]$. These are shown to allow us to compare the uncorrected tracking values with indices which do not incorporate a chance-correction, viz., the FD tracking indices.

Table I contains the estimated values of $\kappa$, the non-parametric FD index (FD I) and the FD index appropriate for polynomial growth curves (FD II) for the three samples along with $( \pm)$ the half-width of the corresponding confidence interval. The significance level for the goodness-of-fit tests was set at 0.05 . It is seen that the three groups all track quite well, no matter which of the indices is considered.

There are, however, some interesting patterns in the results. The most obvious of these is that as one proceeds from left-to-right in the table, i.e. from $x$ to FD I to FD II, the values of the indices increase and the widths of the half-intervals decrease: We appear to be getting more tracking and to be surer of it.

\section{Discussion}

When comparing $\alpha$ with FD I, both of which are unstructured and nonparametric in the sense that neither makes any assumptions concerning the structure of the growth curves nor the distribution of the measurement being considered, it is important to realize that $\kappa$ is chance corrected, i.e. $\kappa$ represents the amount of tracking possible beyond chance which was realized in the data. The uncorrected tracking indices, $P_{0}$, cited earlier, are much more in line with the values of FD I. The fact that the half-widths of the confidence intervals for FD I are roughly $1 / 2$ those for $x$ may

\section{TABLE II}

EFFECTS OF CHANGING $K$ IN $G_{3}$

\begin{tabular}{lllll}
\hline & $K=2$ & $K=3$ & $K=4$ & $K=5$ \\
\hline$P_{\mathrm{o}}$ & 0.89667 & 0.82000 & 0.86333 & 0.61333 \\
$\kappa$ & 0.79328 & 0.72796 & 0.81774 & 0.51625 \\
S.E.( $\boldsymbol{x})$ & 0.05774 & 0.04098 & 0.03334 & 0.02891 \\
Half-interval & 0.11547 & 0.08196 & 0.06668 & 0.05781 \\
\hline
\end{tabular}


just be a product of the particular data sets under consideration, but it should also be realized that $x$ is a relatively complicated ratio of probabilities while FD I represents an average of simple proportions. Note also that the value of $\kappa$ depends on the number, $K$, of tracks specified for the analysis. Those shown in Table I are for $K=3$. An indication of what happens for other values of $K$ is given in Table II where the results for $K=2, K=3, K=4$ and $K=5$ for $G_{3}$ (the high SES Ladinos) are contrasted.

It is seen that the choice of $K$ may substantially effect the value of $\alpha$ and the precision with which it is estimated. We might also note that it is possible - perhaps contrary to expectation - for $\kappa$ to increase with increasing $K(K=2$ vs. $K=4)$.

The comparison of FD I and FD II shows that considerably more tracking is in evidence when FD II is used. One possible reason for this has already been mentioned: Smoothing by fitting polynomials may avoid 'minor crossings' which reflect random fluctuations more than overall growth patterns. Indeed, suppose that two individuals had the same growth curve and departures from that curve were random in nature. FD I would pick up the random crossings thus induced, but FD II would, on the average at least, fit the same polynomials to these individuals and the obvious tracking between them would be preserved. On the other hand, the use of FD II depends on the assumption that polynomials of some degree will be adequate to fit the individual growth curves. While we do provide a test for this, it must be realized that the value of FD II depends on the degree of the polynomial actually fit. In Table I, we used the program-determined $\mathrm{D}$ for each of the groups, viz., $D=1$ for $G_{1}$ and $G_{2}$ and $D=2$ for $G_{3}$. Had we forced $D=1$ in $G_{3}$ (by appropriately choosing the user-specified level of significance for the goodness-of-fit tests in the program), the value of FD II would have been 0.97368 , an even higher value than for $D=2$, despite the fact that $D=1$ was found to not adequately fit the growth profiles in that group. Conversely, fitting a higher-than-necessary degree polynomial will also effect the value of FD II; and this value can be smaller than that based on the lower degree. For example, using $D=2$ in $G_{2}$ results in a FD II value of 0.93158 .

It is obviously difficult (and dangerous) to try to compare the three tracking indices considered in this paper on the basis of their performances on three data sets. While all three indices clearly indicate the existence of tracking behavior within the three groups of Guatemalan schoolchildren, there are subtle differences between these indices and we are not in a position to recommend the use of one over any other. Indeed, we believe that their performance on a wide variety of data sets needs to be studied before any recommendations can be made and it is in this spirit that programs facilitating such comparisons are made available.

The following summary statements may be useful to those interested in making such comparisons. FD I and the $\kappa$ index are unstructured and non-parametric in that neither makes any assumptions about the form of the growth curves nor about the distribution of the measurement under consideration. FD I is based on the the notion of the crossing of growth profiles; kappa is based on the numbers of times individuals are in tracks defined in terms of quantiles. A given pair of individuals may cross a large number of times even if they are always in the same track; or they may have no crosses even though they both exhibit a number of track transitions. Individuals who maintain relative rank and/or tend to stay in a single track may have 
widely differing growth profiles: Neither ensures parallel growth profiles. $\kappa$ depends on the choice of $K$, but has the advantage of being chance-corrected. This correction, however, somewhat complicates the expression of the $\kappa$ statistic and may result in wider confidence intervals than those based on the simpler FD I statistic.

FD II is structured: It is assumed that polynomials may be fit to the individual growth curves. When this assumption is tenable, FD II may be preferable to FD I in that polynomial smoothing may eliminate 'minor crossings' thereby more accurately reflecting the overall course of growth. FD II, however, depends on the degree of the polynomial fit to the individual growth curves and the tests employed to find the minimal $\mathrm{D}$ adequate to fit the data are parametric - they assume that the vector of observations for each individual has a multivariate normal distribution. Given D, the rest of the computations required to produce FD II do not depend on normality, but since this assumption is necessary to determine $\mathrm{D}$ and the value of FD II depends on D, the FD II procedure has an important parametric component.

We note that a FORTRAN program performing the FD analysis is available [7]; however, it is limited to at most third degree polynomials. Additionally, the graphics for this program are accomplished outside of the main program using SYGRAPH [8]. In contrast, our program does not restrict the degree of the polynomial, is menu driven rather than command driven (and therefore very easy to use), directly produces graphics and does not require that the user have additional compilers/interpreters at his or her disposal. For a description of a related approach and further examples, see Ref. 9.

\section{Acknowledgment}

This research was sponsored by grant DE08730 from the National Institute of Dental Research.

\section{Appendix. Computer Implementation}

This program can be obtained on a 5.25 " or $3.5^{\prime \prime}$ diskette by sending $\$ 10$ to defray the cost of handling and licensing fees. Please specify the size and capacity of diskettes when making requests. The progam will run on a IBM-PC/XT or AT compatible computer. The computer must be equipped with a numerical coprocessor from the 8087 family and $640 \mathrm{~K}$ of memory. The computer must be configured so that at least $430 \mathrm{~K}$ of memory is available, ie., not tied up with memory resident programs such as Windows. EGA or VGA graphics capability is required to display the color graphics. No additional software is required (other than what one would normally use to enter a data set); run-time modules are supplied with the program so that no compiler or interpreter is necessary. The program, written in GAUSS, version 2.0, revision 20, requires no additional installation or modification and is run with a single command. When requesting the program, address inquiries to EDS and make checks payable to Baylor College of Dentistry.

\section{References}

1 Schneiderman ED, Willis SM, Kowalski CJ and Ten Have TR: Computation of Foulkes and Davis' nonparametric tracking index using GAUSS, Am J Hum Biol, 4 (1992) 417-420. 
2 Foulkes MA and Davis CE: An index of tracking for longitudinal data, Biometrics, 37 (1981) 439-446.

3 Schneiderman ED, Kowalski CJ and Ten Have TR: A GAUSS program for computing an index of tracking from longitudinal observations, Am J Hum Biol, 2 (1990) 475-490.

4 Schneiderman ED and Kowalski CJ: Implementation of Rao's one-sample polynomial growth curve model using SAS, Am J Phys Anthropol, 67 (1985) 323-333.

5 Bogin B, Sullivan T, Hauspie R and Mac Vean RB: Longitudinal growth in height, weight and bone age of Guatemalan Ladino and Indian school children, Am J Hum Biol, 1 (1989) 103-113.

6 Schneiderman ED, Willis SM, Kowalski CJ and Ten Have TR: A PC program for comparing tracking indices in several independent groups, Am J Hum Biol, 4 (1992) 399-401.

7 Dallal GE: TRACK: A FORTRAN program for computing the Foulkes Davis tracking index, Comput Biol Med, 19 (1989) 367-371.

8 Wilkinson L: SYGRAPH: The System for Graphics, Systat, Evanston IL (1988).

9 Goldstein H: Measuring the stability of individual growth patterns, Ann Hum Biol, 8 (1981) 549-557. 